\title{
Effects of attention on localization of stimuli in the visual field
}

\author{
YEHOSHUA TSAL and TAL BAREKET \\ Tel Aviv University, Tel Aviv, Israel
}

\begin{abstract}
Subjects localized a small letter that appeared inside one of three large circles that was either validly or invalidly cued. The results indicated that, although unattended stimuli were localized fairly accurately, attention still improved localization. Moreover, the results revealed a greater dispersion of localization responses along the horizontal axis and also showed that mislocalization of attended peripheral stimuli is biased toward the periphery.
\end{abstract}

In the present study, the effects of attention on the localization of stimuli at the center and at the periphery of the visual field were investigated. Although the process of localization has been implicated in theorizing about virtually every aspect of visual attention, few studies have directly assessed the effect of directing attention to a given region on the effectiveness of localizing stimuli within that region. In most studies of visual attention, location has been used as part of the manipulation, primarily in the form of a spatial cue, whereas performance related to other dimensions, such as shape, orientation, or color, has been the dependent measure.

Those few studies that have measured localization in addition to identification performance do show that attention produces significant effects on the localization of stimuli in the visual field. Butler (1980) presented a matrix containing nine letters and found that cuing the position of the letter within the matrix reduced errors of mislocation more than it reduced errors of intrusion. $\mathrm{He}$ thus concluded that attention is required primarily for localization and less so for identification. Egly and Homa (1984) investigated the effects of attention on localizing a target that could appear in one of eight possible locations on each of three possible perimeters surrounding fixation. They obtained significant benefits and costs in localization performance when a precue specified the correct or the incorrect perimeter, respectively. Muller and Rabbit (1989) instructed subjects to localize and identify the orientation of the letter $T$, presented in one of four possible locations. They found that valid and invalid peripheral cues produced significant benefits and costs on localization performance, irrespective of whether the target was correctly identified.

Part of this research was presented at the 34th Annual Meeting of the Psychonomic Society, November 1993, in Washington, D.C. This research was supported by Grant 0610710551 from the Israel Academy of Sciences. Correspondence concerning this article should be addressed to Y. Tsal, Department of Psychology, Tel Aviv University, Ramat Aviv, Tel Aviv 69978, Israel (e-mail: jehoshua@freud.tau.ac.il).
The above effects of attention on localization seem to stand in contrast with the demonstrated ability to localize stimuli without attention, as manifested, for example, in the automatic attraction of attention to the general area of an unattended peripheral cue (see, e.g., Jonides, 1981) or in studies showing accurate preattentive location-based judgments when fine localization is not required (e.g., Sagi \& Julesz, 1985).

This apparent inconsistency can be resolved by considering propositions made in recent studies (Cohen \& Ivry, 1989, 1991; Tsal, Meiran, \& Lamy, 1995; Tsal \& Shalev, 1996) that rough localization is possible at the preattentive level, whereas fine localization requires focal attention. The above studies (Butler, 1980; Egly \& Homa, 1984; Muller \& Rabbit, 1989), which showed improved localization with attention, cannot be used to test these recent propositions, because they simply compared number of errors with and without attention. Their results provide no information concerning the metrics of localization performance with and without attention. In other words, they cannot specify the spatial spread of uncertainty that exists at the preattentive level and to what extent it is reduced by the allocation of attentional resources.

Recent indirect but suggestive evidence seems to support the distinction between preattentive rough localization and attentive fine localization. Cohen and Ivry (1989) showed that the magnitude of illusory conjunctionsthat is, incorrectly combining the features of unrelated objects (Treisman \& Schmidt, 1982)-is largely constrained by spatial proximity. When attention is diverted from the display, illusory conjunctions occur only when the items are separated by less than $1^{\circ}$ of visual angle. Separation of greater than $2^{\circ}$, in fact, results in fewer conjunctions than would be expected by chance. In a later study, Cohen and Ivry (1991) demonstrated a similar phenomenon in visual search. They reported that conjunctive search, which is typically characterized by steep serial functions (Treisman \& Gelade, 1980), can be performed preattentively as long as adjacent items in the display are separated by more than $0.78^{\circ}$ of visual angle. The results of these two studies suggest that the spatial uncertainty 
produced by inattention is confined to an area of less than $1^{\circ}$ of visual angle.

In the present study, the subjects were required to localize a briefly presented stimulus that was presented at a cued or an uncued location. The magnitude of dispersion of localization responses around the stimulus served as a measure of localization inaccuracy. The major purpose was to compare these magnitudes for attended and unattended stimuli by employing a variation of the costbenefit method (Posner, 1980). Using the conventional peripheral-cuing technique is inappropriate in the present context, as it would be tantamount to presenting in the target location either two successive stimuli or a single one in the valid and invalid trials, respectively. Hence, improved localization performance in the valid trials might simply be due to the double activation of the same location. To overcome this problem, in the present experiment, the subjects had to localize and identify a single letter that appeared inside one of three large circles. One of the circles abruptly changed its color for a brief duration shortly before the stimulus was presented. On $75 \%$ of the (valid) trials, the letter appeared inside this cued circle, and on $25 \%$ of the (invalid) trials, the letter appeared inside one of the two remaining circles. Accordingly, the positions inside the cued circle were defined as attended, whereas the positions inside the other two circles were defined as unattended.

\section{METHOD}

\section{Subjects \\ The subjects were 8 volunteers ranging in age from 23 to} 33 years. All had normal or corrected-to-normal vision.

\section{Stimuli and Procedure}

Stimulus presentation and data collection were controlled by an IBM PC/AT with a VGA graphic display. A chinrest was used to stabilize viewing distance at $50 \mathrm{~cm}$ so that $1 \mathrm{~cm}$ on the display corresponded to $1.15^{\circ}$ of visual angle. On each trial, the subjects were required to localize and identify a single brown letter that appeared inside one of three large gray outline circles. One of the circles abruptly changed its color for a brief duration, shortly before the stimulus was presented. On $75 \%$ of the (valid) trials, the letter appeared inside this cued circle, and on $25 \%$ of the (invalid) trials, the letter appeared in one of the two remaining circles. Each circle subtended a visual angle of $6.14^{\circ}$ in diameter. One was positioned at the center, and the other two were centered at $9^{\circ}$, one to the right and one to the left of fixation. The target letter was randomly selected from the following set: A, E, Q, W, and Z. Each letter subtended a visual angle of $0.39^{\circ}$ in height and $0.26^{\circ}$ in width. The letter appeared randomly in any possible position inside one of the circles. Each trial consisted of the following sequence of events. First, a fixation field containing the three circles and a small central fixation plus sign appeared for $2 \mathrm{sec}$. The fixation mark then disappeared; one of the circles turned yellow for $50 \mathrm{msec}$ and turned back gray for another $50 \mathrm{msec}$. Then, the letter appeared for $50 \mathrm{msec}$ inside one of the circles. Thus, the duration from the onset of the cue to the offset of the target letter was $150 \mathrm{msec}$. The three circles remained on until the subject responded. The subjects first localized the letter by touching the screen with their preferred hand with a thin marker. The cursor was then moved by the experimenter to the perceived stimulus location, and the response was recorded. Then,
}

the subjects identified the letter by pressing the corresponding key on the extended keyboard. Auditory signals were used to provide feedback for correct identification and for good localization (a deviation smaller than $0.6^{\circ}$ from the stimulus position) on every trial. Each subject was presented with 144 valid trials and 48 invalid trials, which were randomly intermixed. Each of the three circles was cued equally frequently and in random order. On invalid trials, when a given circle was cued, the target letter appeared in either of the noncued circles equally frequently and in random order. The experimental block was preceded by a practice block that included $30-50$ trials.

\section{RESULTS}

Figure 1 presents the distribution of localization responses around the central and peripheral stimuli for the attended and unattended trials. The figure contains the entire set of data - namely, the position of each response for each subject on every trial. In order to concisely describe the magnitude and direction of dispersion, all the stimulus positions of each condition were placed on a single origin (the intersection of the vertical and horizontal axes). The diameter of the circle surrounding each stimulus position designates $1^{\circ}$ of visual angle. It is important to keep in mind that there were three times as many responses for the valid trials as for the invalid ones. Thus, the actual differences in localization dispersion between the attended and the unattended conditions are actually larger than may appear in the figure. The figure suggests that attention improves localization and that this effect is stronger in the center than in the periphery. The figure also shows that responses are more dispersed along the horizontal than along the vertical axis and that the right and left attended peripheral stimuli are most often mislocalized toward the periphery rather than toward the center. Each of these effects is analyzed and discussed below.

The localization data were first converted into deviation scores representing the absolute distances between stimulus locations and the positions of their localization responses. A preliminary analysis showed no difference in the magnitude of localization dispersion of unattended peripheral targets, whether the central or the other, peripheral circle was precued. An overall analysis of variance (ANOVA) of location (central vs. peripheral) $\times$ attention (valid vs. invalid) $\times$ subjects performed on these measures indicated that all the effects were highly significant. Central stimuli were localized more accurately than peripheral ones $[F(1,7)=85.81, p<.0001]$, and attended stimuli were localized more accurately than unattended ones $[F(1,7)=15.14, p=.006]$. The interaction between these two factors was also significant $[F(1,7)=10.80$, $p<.02]$. Analysis of simple effects indicated that the effect of attention was significant only for the central stimuli $[F(1,7)=33.67, p=.007]$.

Given the uneven distribution of responses along the two axes, additional analyses were performed separately on the horizontal and vertical components of the deviation scores. Thus, two distances were computed between each stimulus location and the position of its localization 


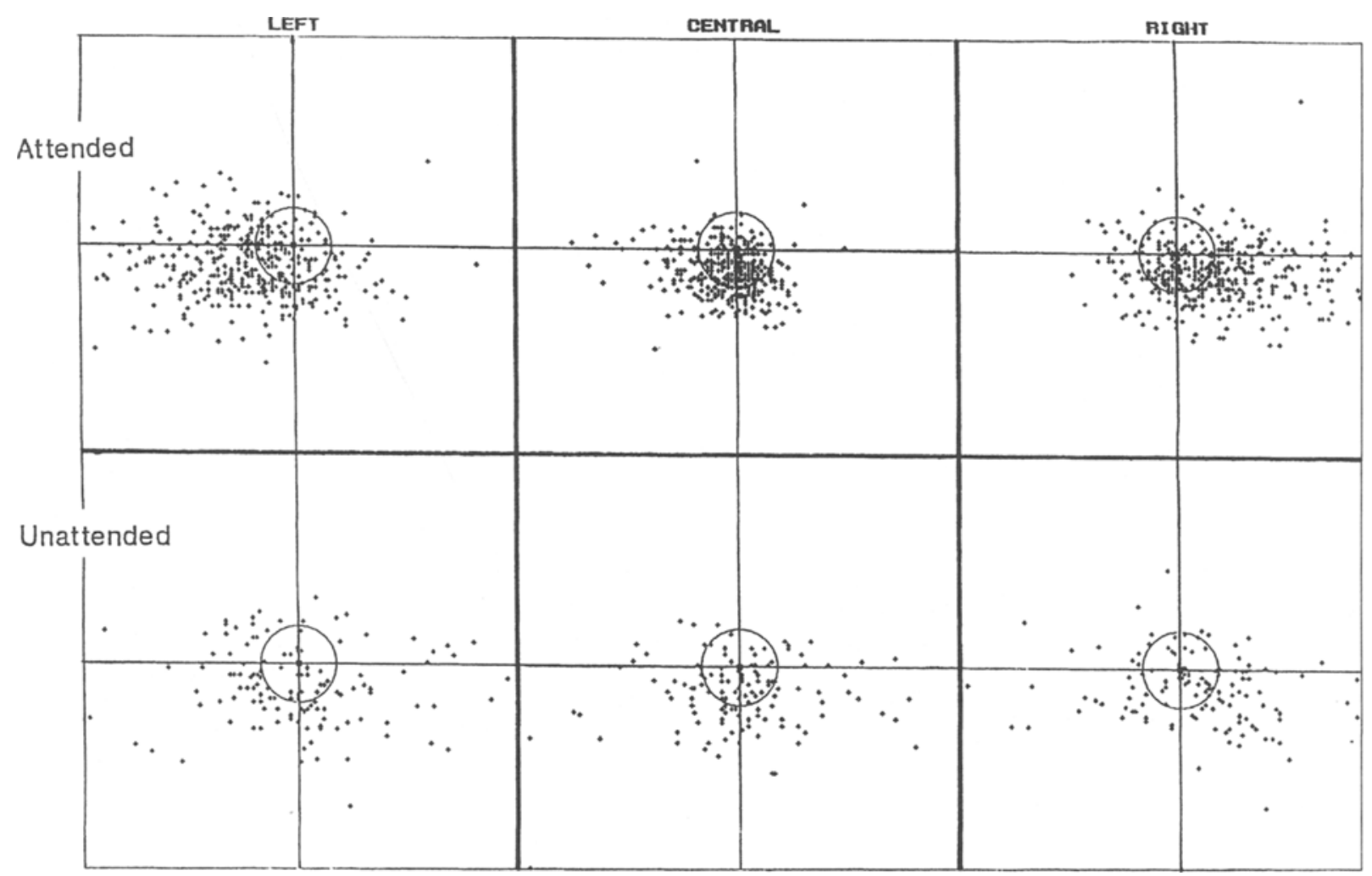

Figure 1. Distributions of localization responses around stimulus positions. The diameter of the circle surrounding each stimulus position designates $1^{\circ}$ of visual angle.

response-the horizontal distance $(x)$ and the vertical distance $(y)$. The mean values of each of these measures represent the average dispersion of localization responses around the stimulus along either the horizontal or the vertical axis. These values are presented in Table 1 . Separate overall ANOVAs were conducted on the horizontal and vertical measures. For the horizontal measure, all the effects were highly significant. Central stimuli were localized more accurately than peripheral ones $[F(1,7)=$ $67.23, p<.0001]$, and attended stimuli were localized more accurately than unattended ones $[F(1,7)=13.7$, $p<.008]$. The interaction between these two factors was also significant $[F(1,7)=12.49, p<.01]$. Analysis of simple effects indicated that the effect of attention was significant only for the central stimuli $[F(1,7)=33.67$, $p=.007]$. For the vertical measures, the two main effects were highly significant. Central stimuli were better localized than peripheral ones $[F(1,7)=9.05, p<.02]$, and attended stimuli were localized better than unattended ones $[F(1,7)=8.69, p<.025]$. The interaction was not significant. Thus, attention affected vertical localization of both central and peripheral stimuli. Overall, the present findings show that attention improves the localization of central stimuli and, to a lesser extent, the localization of peripheral stimuli as well.

Figure 1 and Table 1 suggest that the responses were significantly more dispersed along the horizontal axis, rel- ative to the vertical. A statistical analysis compared the average horizontal and vertical deviations across the three circles and, indeed, confirmed this observation for the attended $[t(7)=17.19, p<.0001]$ as well as the unattended $[t(7)=15.97, p<.0001]$ stimuli.

Figure 1 also suggests that the right and left attended peripheral stimuli were mislocalized further toward the temporal periphery. In order to examine the reliability of this effect, the mean directional displacement was calculated for each subject and submitted to a statistical analysis. The analysis revealed that only the attended stimuli were significantly mislocalized further toward the temporal periphery $[t(7)=14.46, p<.0001$, for the left side, and $t(7)=11.85, p<.0001$, for the right side]

A preliminary analysis showed no difference in the magnitude of identification errors of unattended peripheral targets, whether the central or the other, peripheral circle was precued. An overall ANOVA indicated that central letters were identified more accurately than peripheral ones $[F(1,7)=64, p<.001]$ and that there was a marginally significant tendency to identify the attended letters more accurately than the unattended ones $[F(1,7)=$ $4.24, p=.0785]$. The interaction between eccentricity and attention was not significant. The relatively weak effect of attention on identification responses, which stands in contrast to the results of many studies of visual attention (e.g., Posner, 1980), might possibly be attributed to the 
Table 1

Mean Localization Deviations in

Degrees of Visual Angle (With Standard Deviations)

\begin{tabular}{|c|c|c|c|c|c|}
\hline \multirow[b]{3}{*}{ Location } & \multirow[b]{3}{*}{ Axis } & \multicolumn{4}{|c|}{ Stimuli } \\
\hline & & \multicolumn{2}{|c|}{ Attended } & \multicolumn{2}{|c|}{ Unattended } \\
\hline & & $M$ & $S D$ & $M$ & $S D$ \\
\hline \multicolumn{6}{|l|}{ Central } \\
\hline & Horizontal & 0.33 & 0.1 & 0.60 & 0.19 \\
\hline & Vertical & 0.25 & 0.06 & 0.35 & 0.14 \\
\hline \multicolumn{6}{|l|}{ Peripheral } \\
\hline & Horizontal & 0.72 & 0.24 & 0.75 & 0.24 \\
\hline & Vertical & 0.32 & 0.07 & 0.39 & 0.11 \\
\hline
\end{tabular}

fact that the general area, rather than the specific stimulus location, was cued in advance.

In order to assess the relationship between the localization and the identification of the stimuli, the mean absolute localization deviations were calculated separately for the identified and misidentified letters in each condition. An overall analysis indicated that the identified letters were localized more accurately than the unidentified ones $[t(7)=10.06, p<.0001]$. This finding suggests a strong link between the localization and the identification of the letters appearing within the circles. Although a causal relationship cannot be deduced from the present data, it is reasonable to assume that a better localization of the stimulus facilitated its identification.

\section{DISCUSSION}

The present study clearly shows that attention plays a significant role in the localization of stimuli in the visual field. The effect of attention was stronger at the center than at the periphery; attention affected both horizontal and vertical localization at the center, but only vertical localization at the periphery. However, several factors should be kept in mind in considering this difference. First, directing attention to the peripheral stimuli may have been difficult under the present conditions, inasmuch as the most extreme stimuli appeared at over $12^{\circ}$ to the right or the left in the far periphery. Second, the effects obtained in the present experiment may be an underestimation of the true magnitude of the effects of attention on stimulus localization, because the manipulation of attention was fairly weak. Indeed, unlike most previous studies, the visual cue directed attention not to the specific stimulus location but to the general area within which the stimulus could occur. Third, since localization deviations were measured from stimulus location, the peripheral displacement effect inflated the dispersion of attended stimuli, thus artificially reducing the true difference between attended and unattended localization spread for peripheral stimuli. Indeed, a subsequent analysis that measured the dispersion of localization responses to peripheral stimuli, irrespective of stimulus location, indicated a significantly greater variability for unattended than for attended stimuli.
A distinction ought to be made between two possibly separate effects of attention on localization. The first may be associated with the shift of attention along the cued horizontal axis. That is, the considerable dispersion of localization responses for the unattended trials along the horizontal axis may partly result from locational uncertainty associated with the shift of attention along this axis. Thus, the significant reduction in this dispersion for the attended trials could reflect the elimination (for the central stimuli) or attenuation (for the peripheral stimuli) of these shifts. The second effect of attention is associated with the presence or the absence of attentional resources in the vicinity of the target and, thus, may possibly concern the more inherent difference between the attended and the unattended visual field. Therefore, the significant effects of attention on vertical localization of both central and peripheral stimuli are important in showing an inherent differential spread of localization in the attended and unattended visual fields, both at the center and at the periphery. These findings are consistent with previous studies that demonstrated significant effects of attention on localization (Butler, 1980; Egly \& Homa, 1984; Muller \& Rabbit, 1989; Prinzmetal, Amiri, Allen, \& Edwards, 1998), as well as with distinctions between coarse localization without attention and fine localization with attention (Cohen \& Ivry, 1989, 1991; Prinzmetal et. al., 1998; Tsal et al., 1995).

Although the results discussed above indicate that attention improved localization precision, the pattern of localization responses clearly showed that the absence of attention did not result in "incorrect" responses. That is, inattention did not scatter responses anywhere on the screen but, instead, expanded slightly the area spanned by the localization responses, indicating that coarse localization is possible without attention. The average deviations in localizing unattended stimuli was $0.48^{\circ}$ at the center and $0.78^{\circ}$ at the periphery. Given that the stimulus could appear at any location within a circle, the diameter of which subtended over $6.14^{\circ}$ of visual angle, this pattern of responses is hardly the manifestation of a random distribution of localization responses, but rather reflects the capacity of the system to enable rough localization without attention. Furthermore, this average area spanned by the localization responses around the unattended stimulus matches the area of spatial uncertainty that can be derived from the studies of Cohen and Ivry (1989, 1991), who investigated visual search and illusory conjunctions and found an area of spatial uncertainty under conditions of inattention that subtended less than $1^{\circ}$ of visual angle. One should keep in mind that the assessment of correct and incorrect localization depends on an arbitrary adjustment of a resolution criterion, where, in accord with a very fine criterion, neither unattended nor attended localization responses were correct, whereas in accord with a rough criterion, both attended and unattended responses were correct. The present results suggest that the localization of stimuli receiving differential 
amounts of attention represents different levels on a scale of localization precision. Thus, we propose that the distribution of responses to unattended stimuli reflects the operation of attentional receptive fields (ARFs), within which there is no resolution or spatial differentiation. The notion of ARF was proposed by Tsal and Shalev (1996), who found that unattended vertical lines were perceived as being longer than attended ones. They proposed that the visual field is composed of a grid of ARFs. An ARF can signal the existence of a stimulus within its boundaries to the central processor, but there is no differentiation or resolution within this unit. Hence, when a given line is presented in the visual field, in most cases its end will cross the boundary of an ARF and stimulate an adjacent unit. The latter ARF will be activated once stimulation surpasses a given threshold and will signal a length in correspondence with its size. Since ARFs composing the unattended field are larger than those composing the attended one, the unattended line will be perceived as being longer than the attended one. This notion received further support in a recent study (Shalev \& Tsal, 1999), which showed that inattention produced a tendency to report broken lines as solid ones. The authors concluded that the attended field consists of ARFs that are sufficiently small to distinguish between the line inner ends and the gap, thus preserving the perception of the broken line. The unattended field, on the other hand, is composed of larger ARFs, within which there is no spatial resolution. A partial stimulation of such an ARF by the line inner ends producing the gap signals the activation of the entire ARF, resulting in the perceptual closure of the gap. Thus, the results of the present study, those of Cohen and Ivry $(1989,1991)$, and those of Tsal and Shalev (1996; Shalev \& Tsal, 1999) suggest that different experimental procedures with which different theoretical questions are investigated seem to converge on the same localization unit, within which there is no differentiation of location.

The present experiment also produced an unexpected effect of attention. When attended stimuli appeared in a peripheral circle, there was a strong systematic tendency to mislocalize them further toward the periphery. Although this peripheral displacement effect has not been documented in the attentional literature, there is evidence for a similar effect reported in studies investigating eye movements (e.g., Hershberger, 1987; Honda, 1989; Matin \& Pears, 1965). The general procedure in these studies involves the following steps. Subjects are first presented with a peripheral cue instructing them to move their eyes in a given direction. Then, following a variable interval, a target is presented in one of several possible locations at the same peripheral direction, and the subjects are instructed to localize the target. When the target is presented before the initiation of the saccade, it is consistently localized further toward the periphery. Although this effect has not been discussed in the context of attention, it may well reflect the same peripheral displacement phenomenon observed in the present study. That is, the abrupt onset of the peripheral cue instructing subjects to move their eyes is likely to attract attention to its location, and thus, the perceived displacement of the target that occurs also before the saccade is initiated seems quite similar to the present peripheral displacement effect.

\section{REFERENCES}

Butler, B. (1980). Attention and stimulus localization. Canadian Journal of Psychology, 34, 119-133.

COHEN, A., \& IVRY, R. (1989). Illusory conjunctions inside and outside the focus of attention. Journal of Experimental Psychology: Human Perception \& Performance, 15, 650-663.

Cohen, A., \& Ivry, R. (1991). Density effects in conjunction search: Evidence for a coarse location mechanism of feature integration Journal of Experimental Psychology: Human Perception \& Performance, 17, 891-901.

EGLY, R., \& HomA, D. (1984). Sensitization of the visual field. Journal of Experimental Psychology: Human Perception \& Performance, 10 , 778-793.

HERSHBERGER, W. (1987). Saccadic eye movements and the perception of visual direction. Perception \& Psychophysics, 41, 35-44.

Honda, H. (1989). Perceptual localization of visual stimuli flashed during saccades. Perception \& Psychophysics, 45, 162-174.

JoNiDES, J. (1981). Voluntary vs. automatic control over the mind's eye's movement. In J. B. Long \& A. D. Baddeley (Eds.), Attention and performance IX (pp. 187-204). Hillsdale, NJ: Erlbaum.

Matin, L., \& Pears, D. J. (1965). Visual perception of direction for stimuli flashed during voluntary saccadic eye movement. Science, $148,1485-1488$.

Muller, H. J., \& RabBit, P. M. (1989). Spatial cuing and the relation between the accuracy of "where" and "what" decisions in visual search. Quarterly Journal of Experimental Psychology, 41, 747-773.

PCSNER, M. I. (1980). Orienting of attention. Quarterly Journal of Experimental Psychology, 32, 3-25.

Prinzmetal, W., Amiri, H., Allen, K., \& Edwards, T. (1998). Phenomenology of attention: I. Color, location, orientation, and spatial frequency. Journal of Experimental Psychology: Human Perception \& Performance, 24, 261-282.

SAGI, D., \& Julesz, B. (1985). "Where" and "what" in vision. Science, 228, 1217-1219.

Shalev, L., \& Tsal, Y. (1999). Detecting gaps with and without attention: Further evidence for attentional receptive fields. Manuscript submitted for publication.

Treisman, A., \& Gelade, G. (1980). A feature integration theory of attention. Cognitive Psychology, 12, 97-136.

Treisman, A., \& Schmidt, H. (1982). Illusory conjunctions in the perception of objects. Cognitive Psychology, 14, 107-141.

Tsal, Y., Meiran, N., \& Lamy, D. (1995). Towards a resolution theory of visual attention. Visual Cognition, 2, 313-330.

TSAL, Y., \& SHALEV, L. (1996). Inattention magnifies perceived length: The attentional receptive field hypothesis. Journal of Experimental Psychology: Human Perception \& Performance, 22, 233-243.

(Manuscript received July 31, 1997; revision accepted for publication March 31, 1998.) 